\title{
Virtual Experiments of Heart Valve Tissues
}

\author{
Siyao Huang and Hsiao-Ying S. Huang*
}

\begin{abstract}
The heart valve tissue mainly contains collagen fibers and valve interstitial cells (VICs) and constantly experiences different stress states during cardiac cycles. Due to the anisotropic architecture of collagen fibers and highly inhomogeneous cell population, the mechanical behavior of the heart valve becomes more complicated. It is known that external mechanical stimuli can lead to extracellular matrix (ECM) remodeling, cellular mechanotransduction, cell migration, and collagen synthesis; however, the mechanism of matrix-to-cell stress transfer remains unclear. Current study presents heterogeneously distributed collagen fibers responsible for transmitting forces into cells by an image-based finite element analysis incorporating histological photomicrographs of porcine heart valve tissues. Besides, nonlinear and anisotropic material properties tissue models are incorporated to quantify and visualize the overall stress distributions in heart valve tissues. By establishing an effectively predictive method with new computational tools and by performing virtual experiments on the heart valves, the role of load transmission in heart valves is clarified. The current study completely illustrates the stress distribution around cells and demonstrates the force transmission and reception between cells and matrix in the heart valve tissue. Therefore, our developed image-based finite element models provide new insights not only into clarifying the role of the force transmission and reception between heterogeneously distributed collagen fibers, but also a better understanding of relationships between the mechanical stimuli, cellular mechanotransduction, cell migration, matrix synthesis, and tissue remodeling in heart valves.
\end{abstract}

\section{INTRODUCTION}

During the cycles of systole and diastole, heart valves are passive tissues that persistently open to allow blood to flow forward and close to prevent blood from flowing backward $[1,2]$. The defective structure and improper functions of heart valves directly affect blood supply for circulation or the heart workload. To date, there have been at least 250,000 people suffering heart valve diseases in the United States [3, 4], and the population is rapidly expanding. Tissue engineering is considered as a potential treatment for heart valve replacements, and the distinct difference from conventional replacements is self-acting tissue repair and remodeling [5-7]. Tissue-engineered heart valves in vitro are derived from autologous cells growing on the polymer scaffold and completely biocompatible to overcome defects of rejection of blood type and tissue type. However, it is still

This work is support by Faculty Research and Professional Development Grant at North Carolina State University. Asterisk indicates corresponding author

S. Huang is with the Department of Mechanical and Aerospace Engineering, North Carolina State University, Raleigh, NC 27695 USA (email: shuang4@ncsu.edu).

*HY. S. Huang, is with the Department of Mechanical and Aerospace Engineering, North Carolina State University, Raleigh, NC 27695 USA (email: hshuang@ncsu.edu). unclear how well the tissue-engineered valves would be cultured and how comparable to the behavior of the native heart valves. Therefore, it is important to understand the microstructure and mechanical properties of native heart valves and establish native-like functional parameters to produce tissue equivalents.

Collagen fibers in heart valves are with inhomogeneous microstructure and nonlinear anisotropic properties, and they constantly experience different stress states during cardiac cycles [8-10]. It is well known that collagen fiber alignment and impaction occur in the circumferential direction during heart valves functioning [11-13]. This anisotropic feature of heart valve tissue can resist deformation in the circumferential direction more than in the radial direction Studies have presented nonlinear anisotropic mechanical properties derived by biaxial tension testing as well as quantification of collagen fibers to understand the microstructure response of the heart valve to force loads [1, 11-14]. In the tissue level, the computational simulation of heart valves has been widely applied, given the simplified homogeneous model containing the highly heterogeneous collagen fiber network [15-18]. The finite element analysis (FEA) is a popular method to simulate the soft structure and solve the mechanical problems in the heart valve design. A number of studies have demonstrated linear homogeneous elastic isotropic or anisotropic models used for simulations of the heart valve in stress field, suggesting stress distribution is dependent on collagen fiber distribution [1517]. In the cellular level, the isolated VICs have provided an understanding of sensibility to the mechanical environment, cell-cell or cell-ECM communication, and the ECM maintenance [19-23]. Observations of VICs have clarified biomechanical properties of heart valve generally corresponding with functions of VICs modulated by the mechanical force environment [14, 24-28].

Although the work mentioned above is beneficial to understand mechanical properties of the heart valve, neither the numerical simulation of stress distribution in simplified homogeneous models nor the biomechanical regulation of the isolated VIC can describe the complete behavior of heart valves in a good manner. How external mechanical forces can translate into altered cellular stress states as well as biomechanical regulation in the heart valve remains unclear. In the current study, an image-based automated FEA used for a nonlinear anisotropic model is incorporated to conduct virtual experiments to better visualize the overall stress distributions in porcine heart valve tissues. The current study demonstrates the force transmission and reception between cells and matrix in the heart valve tissue, and it may be expectable to provide new models for better understanding tissue-cell interactions in heart valves as well. 


\section{MATERIALS AND METHODS}

\section{A. Imaged-Based Finite Element Analysis}

A number of parameters are considered to be dependent on microstructural details. Due to the difficulty to describe the microstructure in conventional FEA, simplified models are built to investigate microstructural behavior. Different from conventional FEA software, the Object-Oriented Finite Element (OOF) software developed by the National Institute of Standards and Technology (Gaithersburg, MD) is applied to the analysis of the properties of generic microstructures $[29,30]$. By capturing a digit microstructural picture where heterogeneous collagen fiber architecture and cell population are visualized, OOF can create an image-based FEA model with material properties specified in the selected pixel groups on the image.

OOF is written in a blend of $\mathrm{C}++$ and Python: Python is used heavily for the user interface, while $\mathrm{C}++$ is reserved for performance-critical code blocks, especially those that tend to be computationally intensive. The study builds two code extensions (discrete packets of code that can dynamically interact with a host program as it executes) that, integrated with the current OOF code, facilitate an automated finite element analysis for heart valve tissues. The extensions are discussed as follows: (1) a pixel selection extension and (2) a collagen fiber material properties extension.

When OOF solves a finite element problem, it is necessary to have all the pixels in an image assigned to a particular pixel group and thereby associated with particular material properties. Should any pixels be missed, the results of the FEA include "holes" where no any material properties are assigned. In order to ensure that all pixels in the images are assigned to a pixel group, a pixel selection extension is developed. The extension uses the properties of the colors in the image to distinguish between various objects in the image. The hue-saturation-intensity (HSI) color space represents colors as a combination of items as following: (1) the shade of a color (i.e., hue), such as blue, green, red, yellow, etc. (2) The vibrancy of the color (i.e., saturation); colors with a low saturation appear to be pale or "washed out." For example, a red hue with a reduced saturation is pink. (3) The intensity of the color, which refers to the brightness of the color, with zero intensity representing fully dark (i.e., black). In photomicrographs of hematoxylin and eosin (H\&E) stained heart valve tissue, it has been readily observed that VIC nuclei (stained dark blue by hematoxylin) are associated with a low intensity (compared to voids appearing as transparent regions in the photomicrograph), while collagen fibers manifest a relatively high saturation. The extension is developed and implemented in two parts: (a) the courier (written in $\mathrm{C}++$ ) iterates through the entire image pixels and uses the comparator to compare the value of the component to the threshold. Once the comparison is true, that pixel is added to the selection. (b) The second part is written in Python and receives values from the user interface for use in constructing the courier. The extension helps ensure that all pixels in the images are assigned to their appropriate pixel groups and the function facilitates material property assignments in the later finite element analysis process.
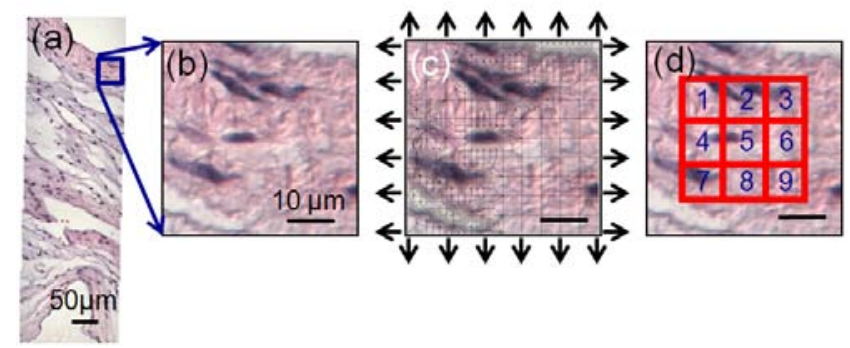

Figure 1. Virtual experiments on pulmonary heart valve tissue histological photomicrographs. (a) An en-face tissue photomicrograph (H\&E stained). (b) A section of interest. (c) Applying boundary conditions on the meshed image. (d) Obtaining calculated stress fields in nine regions on the image. Scale bar $=10 \mu \mathrm{m}, \mathrm{x} 400$.

\section{B. Virtual Experiments on Heart Valve Tissue Histological Photomicrographs}

The virtual experiments employ finite element methods to simulate physiologically relevant biaxial stretching states on tissues and depict changes in collagen fiber orientations and the resultant cellular stress environment (Fig. 1). The porcine pulmonary valve tissue is prepared with H\&E stains; photomicrographs are obtained via an optical microscope. The en-face pulmonary valve leaflet (400x) is used for the stretching simulation with applied external $30 \%$ of strain (Fig. 1c). Histological photomicrographs capturing collagen fibers microstructure and cell morphology are incorporated into the FEA. Due to the heterogeneous distribution and undulated morphology of collagen fibers, anisotropic and nonlinear material properties are features of heart valve tissues. The piecewise linear elastic anisotropic material properties under different strain stretching are published somewhere else [31] (Table 1) and are used to transformed into orthotropic stiffness matrices [32]. Therefore, three stiffness matrices of the pulmonary heart valve tissue are as follows: $0-18 \%$ strain: $\mathrm{C}_{11}=14 \mathrm{kPa}, \mathrm{C}_{12}=\mathrm{C}_{21}=7 \mathrm{kPa}, \mathrm{C}_{22}=16$ $\mathrm{kPa}, \mathrm{C}_{66}=4 \mathrm{kPa} ; 18-28 \%$ strain: $\mathrm{C}_{11}=418 \mathrm{kPa}, \mathrm{C}_{12}=\mathrm{C}_{21}=23$ $\mathrm{kPa}, \mathrm{C}_{22}=51 \mathrm{kPa}, \mathrm{C}_{66}=61 \mathrm{kPa} ; 28-35 \%$ strain: $\mathrm{C}_{11}=1,493$ $\mathrm{kPa}, \mathrm{C}_{12}=\mathrm{C}_{21}=79 \mathrm{kPa}, \mathrm{C}_{22}=176 \mathrm{kPa}, \mathrm{C}_{66}=217 \mathrm{kPa}$. In all these three strain zones, $v_{12}=0.45$ is used for collagen fibers, and it is determined via $-v_{i j} / E_{i}=-v_{j i} / E_{j}$ [32] [33]. Cells and voids are assumed to be isotropic materials $\left(\mathrm{E}_{\text {cell }}=0.9\right.$ $\mathrm{kPa}$ and $v_{\text {cell }}=0.45 ; \mathrm{E}_{\text {void }}=0.01 \mathrm{kPa}$ and $v_{\text {void }}=0.45$ ) [14]. To illustrate the importance of adopting anisotropic materials property, in addition to the heterogeneous distributed collagen fibers, the current study also provides another set of virtual experiment results by using isotropic material properties in collagen fibers: $\mathrm{E}=\mathrm{E}_{\mathrm{x}}=\mathrm{E}_{\mathrm{y}}=408 \mathrm{kPa}, \mathrm{v}=0.45$.

TABLE I. MODULUS OF VALVULAR TISSUES AT VARIOUS STRAINS

\begin{tabular}{|c|c|c|c|}
\hline Modulus E & $\begin{array}{l}0-18 \% \text { of } \\
\text { Strain }\end{array}$ & $\begin{array}{c}18-28 \% \text { of } \\
\text { Strain }\end{array}$ & $\begin{array}{c}28-35 \% \text { of } \\
\text { Strain }\end{array}$ \\
\hline AV C-dir. (kPa) & $89.1 \pm 4.63$ & $825.11 \pm 29.19$ & $1577.17 \pm 53.69$ \\
\hline AV R-dir. (kPa) & $33.94 \pm 1.37$ & $116.43 \pm 4.15$ & $324.93 \pm 9.84$ \\
\hline PV C-dir. (kPa) & $11.31 \pm 0.79$ & $408.23 \pm 18.34$ & $1457.19 \pm 58.1$ \\
\hline PV R-dir. (kPa) & $11.67 \pm 0.61$ & $50.16 \pm 2.01$ & $172.44 \pm 5.24$ \\
\hline
\end{tabular}

\section{RESUlTS AND DisCUSSION}

The FEA results depict the distinct feature of the inhomogeneous collagen fiber microstructure illustrating on 
how mechanical stresses are transferred to cells under biaxial stretching. The region 5 from Fig. 1 is selected to represent the mechanical interaction between ECM and cells (Fig. 2). In the isotropic model, there is a difference between stress values in the circumferential direction and the radial direction: $\sigma_{\mathrm{xx}}=2231.11 \mathrm{kPa}$ and $\sigma_{\mathrm{yy}}=967.82 \mathrm{kPa}$, respectively. The stress distribution is not uniform in this isotropic model due to the inhomogeneous microstructure of the heart valve tissue. Though the material property plays an important role to affect the stress field of the valve tissue, the mechanical stress transmitting from the ECM to cells may not be corresponding well with the assumption of isotropy (Fig. 2a and $2 \mathrm{~b}$ ). The unanticipated results are caused by the effects of the collagen fiber orientation and the distributions or shapes of cells, rather than the material property alone.

In contrast, results from the anisotropic FEA models reveal the mechanisms of how stresses are transferred from ECM to cells. Circled locations (Fig. 2c and 2d) have the same coordinates as ones with arrows in Fig. $2 \mathrm{a}$ and $2 \mathrm{~b}$. Results show that $\sigma_{x x}=978.68 \mathrm{kPa}$ and $\sigma_{y y}=84.06 \mathrm{kPa}$ in Fig. $2 \mathrm{c}$ and $2 \mathrm{~d}$, respectively. It is suggested that the anisotropic material property plays a significant role in how ECM stresses are transferred into cells. In addition, differences between the stress distribution in the circumferential and radial directions are clear in the anisotropic models, whereas isotropic models exhibit similar manners in the stress distribution in the circumferential and radial directions. Nevertheless, the anisotropic property mitigates stress concentrations and leads to lower stress fields in heart valve tissues. Moreover, most local peak stresses observed in both directions occur around cells. Since cells are generally more compliant than ECM, such higher stress values may be mediated by VICs for biomechanical regulations.

A number of FEA studies utilized elastic isotropic or anisotropic materials, and their results suggest that the stress distribution is highly dependent on collagen fiber distribution [15-18]. However, the homogeneous collagen fiber distributions generally adopted in these studies are excluding any cells in the models. Here, we demonstrate a different approach by incorporating both of the collagen fiber architecture and nonlinear anisotropic material property, and we further discuss how the aforementioned properties affect on stress fields of cells inside ECM. In addition, current study provides insights into alterations in heart valve microstructure, mechanical properties, and other potential biomechanical regulations resulting from heart valve diseases, which are caused by the disrupted tissue homeostasis under a variety of pathological conditions [3437].

It is known that the internal inhomogeneous composition, such as the cell distribution, has a great influence on the mechanics of the heart valve, like the deformation of VICs $[14,38]$. VICs are regarded as a regulator associated with synthesizing and maintaining the valvular ECM to provide a structure framework due to the unique profiles of cell-cell and cell-ECM adhesion molecule expression [21, 39] as well as the observation that age-related decreases in VIC numbers accompany collagen fiber degeneration. The abilities of VICs to communicate with each other, with the ECM, and to respond to their environment basically support the mechanisms that allow heart valves to function in an optimal and efficient manner [23]. Furthermore, structuremechanics-property relations are tightly coupled in the biomechanical regulation of the tissue homeostasis induced by the alterations in cellular phenotype and morphology [3437]. It has been observed that VICs are sensible to the mechanical environment, and the VICs' phenotypes and functions can be changed to mediate the tissue microstructure by mechanical stimuli [14, 24-28], which suggests that ECM and cell functions are modulated by mechanical forces and stretches. Tissues respond to the mechanical environment by growth and remodeling, repair, and degradation, and cells provide adaptive responses, such as the synthesis and secretion of cytokines, growth factors, ECM and differentiation to other phenotypes. To response to inappropriate stimuli, VICs may participate in processes that lead to valve degeneration and calcification. It is also important to understand the complex physiology and pathology of VICs and biomechanical regulation of valve function by VICs from the cell level to the tissue level.

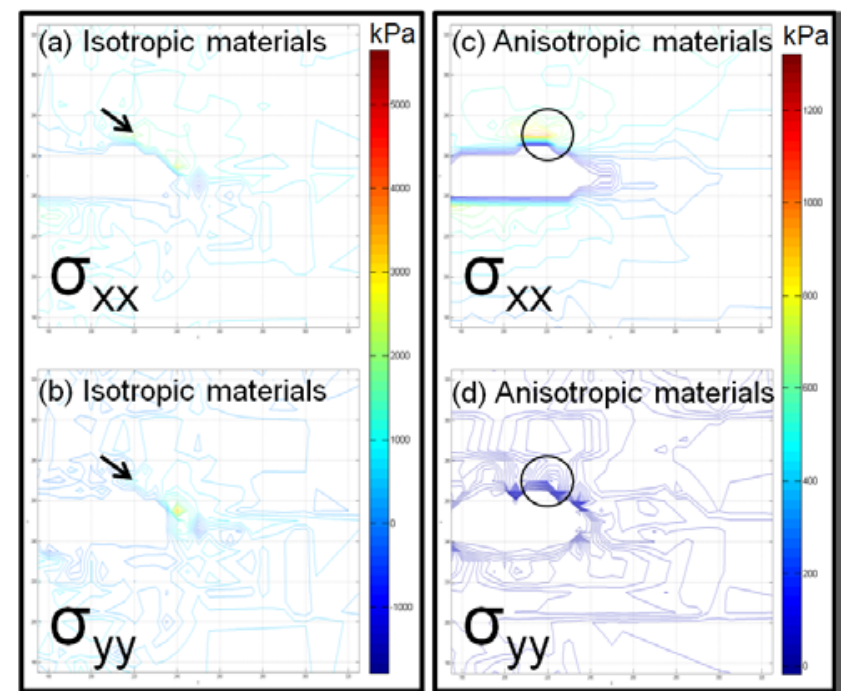

Figure 2. Stress distribution in region 5 of Fig. 1 via virtual experiments under 30\% equal-biaxial stretching. (a) and (b) FEA results with isotropic materials properties. Arrow: $\sigma_{\mathrm{xx}}=2231.11 \mathrm{kPa}$ and $\sigma_{\mathrm{yy}}=967.82 \mathrm{kPa}$ ). (c) and (d) FEA results with anisotropic materials properties. Circled: $\sigma_{x x}=978.68$ $\mathrm{kPa}$ and $\sigma_{\mathrm{yy}}=84.06 \mathrm{kPa}$. The result indicates anisotropic materials property play a significant role in how ECM stress is transferred into cells.

\section{CONCLUSIONS}

To develop native-like functional parameters to produce tissue equivalents in the field of the heart valve repair, it is important to understand the microstructure and material properties of native heart valves, as well as the mechanism of cellular mechanical reaction to the ECM. By using computational tools, the simulation provides a good estimate for better understanding the biological system. A great number of studies have demonstrated and clarified the unique behavior of the heart valve by simulations of simplified homogenous models or analyses with isolated cells in the ECM. Nevertheless, the tissue in reality includes numerous embedded VICs (inhomogeneity) and specific fiber orientation (nonlinear anisotropy materials). The purpose of this study is concerned with the investigation of cell-matrix 
mechanical interactions in heart valves and provides a better understanding of the relationships between the mechanical stimuli and cellular mechanotransduction, cell migration, matrix synthesis, and tissue remodeling in the heart valves. By establishing the effectively predictive method with new computational tools and performing virtual experiments on the heart valves, the role of load transmission in heart valves is clarified. The results clearly indicate that the matrix is more compliant in the radial direction and heterogeneously distributed collagen fibers are responsible for transmitting forces into cells, especially in the circumferential direction. The stress distribution around cells is completely illustrated. These findings will benefit to comprehend fundamentals of structure-mechanics-property reaction as well as the biomechanical regulation mediating cell-matrix interactions. In addition, the results of the proposed work will be practical to clarify or evaluate the relation of the tissue microarchitecture and load transmission in a variety of tissue growth.

\section{REFERENCES}

[1] M.S. Sacks, F.J. Schoen, and J.E. Mayer, Jr., "Bioengineering Challenges for Heart Valve Tissue Engineering," ANNUAL REVIEW OF BIOMEDICAL ENGINEERING, vol. 11, (no. Journal Article), pp. 289-313, 2009.

[2] M.S. Sacks, W.D. Merryman, and D.E. Schmidt, "On the biomechanics of heart valve function," Journal of Biomechanics, vol. 42, (no. 12), pp. 1804-1824, 2009.

[3] A. American Heart, "Heart Disease and Stroke Statistics,"2010.

[4] L. National Heart and I. Blood, "Heart and Vascular Diseases,"2010

[5] G. El Khoury, J.L. Vanoverschelde, D. Glineur, F. Pierard, R.R. Verhelst, J. Rubay, J.C. Funken, C. Watremez, P. Astarci, V. Lacroix, A. Poncelet, and P. Noirhomme, "Repair of bicuspid aortic valves in patients with aortic regurgitation," Circulation, vol. 114, pp. I610-I616, 2006.

[6] R. El Oakley, P. Kleine, and D.S. Bach, "Choice of prosthetic heart valve in today's practice," Circulation, vol. 117, (no. 2), pp. 253-256, Jan 152008.

[7] K. Mendelson and F.J. Schoen, "Heart valve tissue engineering: Concepts, approaches, progress, and challenges," Annals of Biomedical Engineering, vol. 34, (no. 12), pp. 1799-1819, Dec 2006.

[8] G.W. Christie and B.G. Barratt-Boyes, "Mechanical Properties of Porcine Pulmonary Valve Leaflets: How Do They Differ from Aortic Leaflets?," Ann Thorac Surg, vol. 60, (no. 0003-4975), pp. S195-S199, 1995.

[9] K.L. Billiar and M.S. Sacks, "Biaxial mechanical properties of the natural and glutaraldehyde treated aortic valve cusp - Part I: Experimental results," Journal of Biomechanical EngineeringTransactions of the Asme, vol. 122, (no. 1), pp. 23-30, 2000.

[10]P. Stradins, R. Lacis, I. Ozolanta, B. Purina, V. Ose, L. Feldmane, and V. Kasyanov, "Comparison of biomechanical and structural properties between human aortic and pulmonary valve," European journal of cardio-thoracic surgery : official journal of the European Association for Cardio-thoracic Surgery, vol. 26, (no. 3), pp. 634-639, 2004.

[11]M.S. Sacks, D.B. Smith, and E.D. Hiester, "The aortic valve microstructure: Effects of transvalvular pressure," Journal of Biomedical Materials Research, vol. 41, (no. 1), pp. 131-141, 1998.

[12]E.M. Joyce, J. Liao, F.J. Schoen, J.E. Mayer, Jr., and M.S. Sacks, "Functional Collagen Fiber Architecture of the Pulmonary Heart Valve Cusp RID F-3703-2011," Annals of thoracic surgery, vol. 87, (no. 4), pp. 1240-1249, 2009.

[13]M.A.J. Cox, J. Kortsmit, N. Driessen, C.V.C. Bouten, and F.P.T. Baaijens, "Tissue-Engineered Heart Valves Develop Native-like Collagen Fiber Architecture," Tissue Engineering Part a, vol. 16, (no. 5), pp. 1527-1537, 2010.

[14]H.-Y.S. Huang, J. Liao, and M.S. Sacks, "In-situ deformation of the aortic valve interstitial cell nucleus under diastolic loading," Journal of Biomechanical Engineering, vol. 129, pp. 1-10, 2007.

[15]J. Li, X.Y. Luo, and Z.B. Kuang, "A Nonlinear anisotropic Model for Porcine Aortic Heart Valves," Journal of Biomechanics, vol. 34, (no. Journal Article), pp. 1279-1289, 2001.
[16]X.Y. Luo, W.G. Li, and J. Li, "Geometrical stress-reducing factors in the anisotropic porcine heart valves RID B-2824-2011," Journal of Biomechanical Engineering-Transactions of the Asme, vol. 125, (no. 5), pp. 735-744, 2003.

[17]H. Mohammadi, F. Bahramian, and W. Wan, "Advanced modeling strategy for the analysis of heart valve leaflet tissue mechanics using high-order finite element method," Medical engineering \& physics, vol. 31, (no. 9), pp. 1110-1117, 2009.

[18]T.M. Koch, B.D. Reddy, P. Zilla, and T. Franz, "Aortic valve leaflet mechanical properties facilitate diastolic valve function RID C-33862009," Computer methods in biomechanics and biomedical engineering, vol. 13, (no. 2), pp. 225-234, 2010.

[19]D.L. Mulholland and A.I. Gotlieb, "Cardiac valve interstitial cells: Regulator of valve structure and function," Cardiovascular Pathology, vol. 6, (no. 3), pp. 167-174, 1997.

[20]P.M. Taylor, P. Batten, N.J. Brand, P.S. Thomas, and M.H. Yacoub, "The cardiac valve interstitial cell," Int J Biochem Cell Biol, vol. 35, (no. 2), pp. 113-8, 2003.

[21]N. Latif, R. Sarathchandra, R.M. Taylor, J. Antoniw, and M.H. Yacoub, "Molecules mediating cell-ECM and cell-cell communication in human heart valves," Cell biochemistry and biophysics, vol. 43, (no. 2), pp. 275-287, 2005.

[22]A.C. Liu, V.R. Joag, and A.I. Gotlieb, "The emerging role of valve interstitial cell phenotypes in regulating heart valve pathobiology," American Journal of Pathology, vol. 171, (no. 5), pp. 1407-1418, 2007.

[23]A.H. Chester and P.M. Taylor, "Molecular and functional characteristics of heart-valve interstitial cells," Philosophical Transactions of the Royal Society B-Biological Sciences, vol. 362, (no. 1484), pp. 1437-1443, 2007.

[24]D. Choquet, D.P. Felsenfeld, and M.P. Sheetz, "Extracellular matrix rigidity causes strengthening of integrin-cytoskeleton linkages," Cell, vol. 88, (no. 1), pp. 39-48, 1997.

[25]J.E. Schwarzbauer, "Cell migration: May the force be with you," Current Biology, vol. 7, (no. 5), pp. R292-R294, 1997.

[26]M.P. Sheetz, D.P. Felsenfeld, and C.G. Galbraith, "Cell migration: Regulation of force on extracellular-matrix-integrin complexes," Trends in cell biology, vol. 8, (no. 2), pp. 51-54, 1998.

[27]J. Atance, M.J. Yost, and W. Carver, "Influence of the extracellular matrix on the regulation of cardiac fibroblast behavior by mechanical stretch," Journal of cellular physiology, vol. 200, (no. 3), pp. 377-386, 2004.

[28]E. Puklin-Faucher and M.P. Sheetz, "The mechanical integrin cycle," Journal of cell science, vol. 122, (no. 2), pp. 179-186, 2009.

[29]S.A. Langer, E. Fuller, and W.C. Carter, "OOF: An image-based finiteelement analysis of material microstructures," Computing in Science and Engineering, vol. 3, (no. 3), pp. 15-23, 2001.

[30]A.C.E. Reid, R.C. Lua, R.E. Garcia, V.R. Coffman, and S.A. Langer, "Modelling Microstructures with OOF2," International Journal of Materials \& Product Technology, vol. 35, (no. 3-4), pp. 361-373, 2009 2009

[31]H.-Y.S. Huang, B.N. Balhouse, and S. Huang, "Application of Simple Biomechanical and Biochemical Tests to Heart Valve Leaflets: Implications for Heart Valve Characterization and Tissue Engineering," Journal of Engineering in Medicine, (accepted).

[32]W.S. Slaughter, The Linearized Theory of Elasticity, Boston: Birkhauser, 2001.

[33]J.F. Nye, Physical properties of crystals, their representation by tensors and matrices: Oxford, Clarendon Press, 1957, 1957.

[34]P.K. Mays, J.E. Bishop, and G.J. Laurent, "Age-Related-Changes in the Proportion of Type-i and Type-Iii Collagen," Mechanisms of ageing and development, vol. 45, (no. 3), pp. 203-212, 1988.

[35]Y.C. Fung, Biomechanics: mechancial properties of living tissues, New York: Springer Verlag, 1993.

[36]A.D. Durbin and A.I. Gotlieb, "Advances towards understanding heart valve response to in injury," Cardiovascular Pathology, vol. 11, (no. 2), pp. 69-77, 2002.

[37]C. Fayet, M.P. Bendeck, and A.I. Gotlieb, "Cardiac valve interstitial cells secrete fibronectin and form fibrillar adhesions in response to injury," Cardiovascular Pathology, vol. 16, (no. 4), pp. 203-211, 2007.

[38]H.M. Langevin, K.N. Storch, R.R. Snapp, N.A. Bouffard, G.J. Badger, A.K. Howe, and D.J. Taatjes, "Tissue stretch induces nuclear remodeling in connective tissue fibroblasts," Histochemistry and cell biology, vol. 133, (no. 4), pp. 405-415, 2010.

[39]N. Latif, P. Sarathchandra, P.M. Taylor, J. Antoniw, N. Brand, and M.H. Yacoub, "Characterization of molecules mediating cell-cell communication in human cardiac valve interstitial cells," Cell Biochem Biophys, vol. 45, (no. 3), pp. 255-64, 2006. 